\title{
Hypergravity-induced plastic alteration of the vestibulo-sympathetic reflex involves decrease in responsiveness of CAMK2-expressing neurons in the vestibular nuclear complex
}

\author{
Chikara Abe $^{1}$ D $\cdot$ Yusuke Yamaoka $^{1} \cdot$ Yui Maejima ${ }^{1} \cdot$ Tomoe Mikami $^{1} \cdot$ Hironobu Morita $^{1}$
}

Received: 8 July 2019 / Accepted: 9 August 2019 / Published online: 21 August 2019

(c) The Author(s) 2019

\begin{abstract}
The vestibular system contributes to not only eye movement and posture but also the sympathetic response. Plastic alteration of the vestibulo-sympathetic reflex is induced by hypergravity load; however, the mechanism remains unknown. Here, we examined $2 \mathrm{~g}$-induced changing in responsiveness of CAMK2-expressing neurons in the vestibular nucleus complex using optogenetic tools. The excitatory photostimulation of the CAMK2-expressing neurons in the unilateral vestibular nuclear complex induced body tilt to the contralateral side, while inhibitory photostimulation showed the opposite response. Photoactivation of either cell body or the axonal terminal in the rostral ventrolateral medulla showed sympathoexcitation followed by the pressor response. Furthermore, this response was significantly attenuated $(49.8 \pm 4 \%)$ after the 1 st day of $2 \mathrm{~g}$ loading, and this value was further reduced by the 5th day $(22.4 \pm 3 \%)$, suggesting that $2 \mathrm{~g}$-induced attenuation of the vestibulo-sympathetic reflex involves at least decrease in responsiveness of CAMK2-expressing neurons in the vestibular nuclear complex.
\end{abstract}

Keywords Gravity $\cdot$ Vestibule $\cdot$ Plasticity $\cdot$ Arterial pressure $\cdot$ Optogenetics $\cdot$ Viral vector

\section{Introduction}

The peripheral gravity sensor, located in the inner ear, consists of two components, namely, the semicircular canals and otolith organs, which detect angular and linear accelerations, respectively. The signals from each sensor are transmitted to the brain, specifically to the vestibular nuclear complex (VNC), through the vestibular nerve [1]. The vestibular system contributes to both eye movement (i.e., the vestibulo-ocular reflex) and posture (i.e., the vestibulospinal reflex), and is important for the understanding of the body's dynamics and kinematics [2]. The vestibular system also participates in the sympathetic nervous response (vestibulo-sympathetic reflex) [3, 4]. Acute gravitational

Electronic supplementary material The online version of this article (https://doi.org/10.1007/s12576-019-00705-5) contains supplementary material, which is available to authorized users.

Chikara Abe

chikara@gifu-u.ac.jp

1 Department of Physiology, Gifu University Graduate School of Medicine, 1-1 Yanagido, Gifu 501-1194, Japan change or galvanic vestibular stimulation (GVS) increases the sympathetic nerve activity in rodents and human subjects [5-7]. The vestibulo-sympathetic reflex may contribute to orthostatic tolerance, which is typically associated with the baroreflex $[8,9]$. Furthermore, recent studies have shown that the vestibular system is involved in other physiological functions; muscles and bone [10], food intake [11], circadian rhythm [12], and body temperature regulation [13].

The vestibular system is one of the plastic organs which is easily affected by chronic gravitational changes [14]. The studies using astronauts have reported the decrease in sensitivity of vestibulo-ocular [15], vestibulo-spinal [16], and vestibulo-sympathetic (cardiovascular) reflexes [9, 17], which increase the risk of falls and bone fractures. In addition to microgravity, we demonstrated that these plastic alterations in the vestibulo-sympathetic reflex also occur in rats exposed to hypergravity $[5,18,19]$. When rats were maintained in a hypergravity environment, the sympathetic and cardiovascular responses induced by vestibular stimulation, including free drop and linear acceleration, were attenuated. Although we clarified that attenuation of the vestibulo-sympathetic reflex is due to a decrease in head movement-related phasic inputs to the peripheral vestibular 


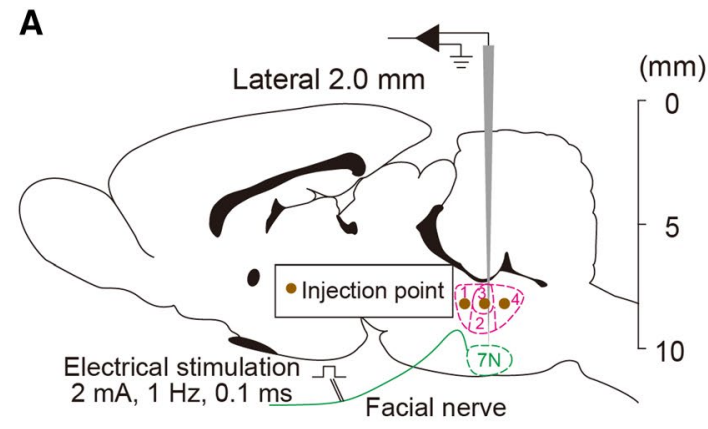

1. Superior vestibular nucleus, 2. Medial vestibular nucleus 3. Lateral vestibular nucleus, 4 . Spinal vestibular nucleus

Fig. 1 a Injection of viral vector to express either ChR2 or eArchT in the CAMK2-expressing neurons in the vestibular nucleus complex (VNC). The facial nucleus, which is found through the evoked potential induced by electrical stimulation of the facial nerve, is regarded

organs $[20,21]$, the mechanism of the plastic alteration is still unclear.

The morphological and genetic changes in the peripheral vestibular organ are induced by the gravitational change. Exposure to microgravity for 9 days significantly increased total ribbon synapses and sphere-like ribbons in type I and II hair cells in rats [22]. On the other hand, hypergravity load for 1 week showed a decrease in GluR2 mRNA expression in the vestibular ganglion [23]. These findings indicate that gravitational change might influence the responsiveness of neurons in the VNC because these neurons are downstream of the vestibular pathway. Indeed, NR1 mRNA expression in the VNC was decreased by hypergravity load [23]. If this is true, activation of neurons in the VNC-induced sympathetic response might be attenuated in rats which are maintained in the hypergravity environment. To test this hypothesis, we focused on the functional change of the putative excitatory neuron, calcium/calmodulin-dependent protein kinase II (CAMK2)-expressing neurons, in the VNC induced by chronic 2 g loading, and then evaluated whether the sympathoexcitation induced by optogenetic stimulation of these neurons was attenuated.

\section{Materials and methods}

\section{Animals}

The animals used in the present study were maintained in accordance with the "Guiding Principles for Care and Use of Animals in the Field of Physiological Science", set by the Physiological Society of Japan. The experiments were approved by the Animal Research Committees of Gifu University. Male $(n=38)$ and female $(n=32)$ rats, weighing 220-280 g, were purchased from Chubu Kagaku Shizai.
B

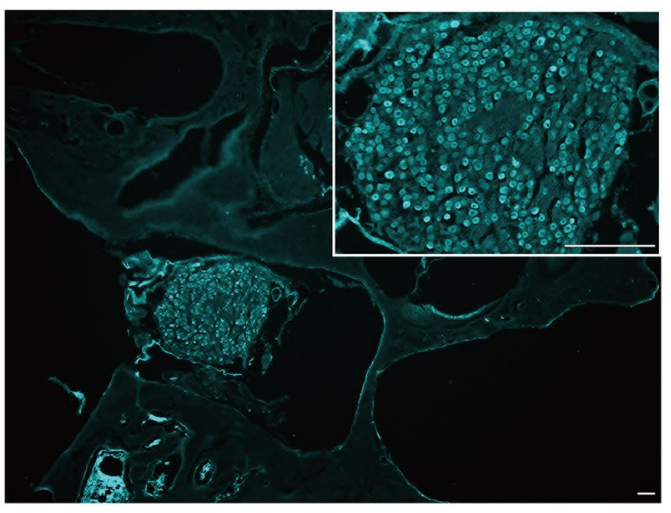

as a landmark to find the VNC. b Neuronal retrograde tracer (FluoroGold) was injected at the left VNC. Fluoro-gold expression was observed in the vestibular ganglion. Scale bar: $100 \mu \mathrm{m}$ for main panels and $200 \mu \mathrm{m}$ for insets

\section{Anesthesia and postoperative management}

All surgeries were conducted under aseptic conditions, and rats were anesthetized with a mixture of medetomidine hydrochloride $(0.3 \mathrm{mg} / \mathrm{kg})$, midazolam $(4 \mathrm{mg} / \mathrm{kg})$, and butorphanol tartrate via the intraperitoneal administration (i.p.) route $(5 \mathrm{mg} / \mathrm{kg})$. The depth of anesthesia was deemed sufficient when the corneal and hind-paw withdrawal reflexes were absent. Additional anesthetic was administered as necessary ( $10 \%$ of the original dose, i.p.). Body temperature was maintained at $37.0 \pm 0.5^{\circ} \mathrm{C}$ with a servo-controlled temperature pad. After surgery, rats received postoperative boluses of atipamezole (an $\alpha 2$-adrenergic antagonist, $2 \mathrm{mg} / \mathrm{kg}$ ), penicillin $\mathrm{G}$ potassium $(3000 \mathrm{U} / \mathrm{kg}$ ), and ketoprofen $(4 \mathrm{mg} / \mathrm{kg})$ via the subcutaneous administration (s.c.) route. Rats were solely housed to maintain the ferrule on the head. Rats were under a 12:12-h light-dark cycle. The room temperature was maintained at $24 \pm 1{ }^{\circ} \mathrm{C}$.

\section{Injection of the viral vectors into the VNC and optical fiber placement}

AAV2-CaMK2a-hChR2(H134R)-mCherry, AAV2CaMK2a-eArchT3.0-EYFP, and AAV2-CaMK2a-mCherry were purchased from the University of North Carolina vector core [obtained courtesy of K. Deisseroth (Stanford University)]. These viral vectors were injected unilaterally into the left VNC, followed by the placement of an optical fiber (Fig. 1a). Using a rat brain atlas, the VNC was located at the dorsal side from the facial motor nucleus. The left mandibular branch of the facial nerve was revealed through a small skin incision for successive electrical stimulation. Thereafter, the rat was placed prone on a stereotaxic apparatus (SR-6M-HT, Narishige). The viral vector was loaded into a 
$1.2 \mathrm{~mm}$ internal diameter glass pipette broken to a $25 \mu \mathrm{m}$ tip (external diameter) and introduced into the brain through a $1.5 \mathrm{~mm}$ diameter hole drilled into the occipital plate caudal to the parieto-occipital suture on the left side. The facial nerve was then stimulated $(0.1 \mathrm{~ms}, 1-300 \mu \mathrm{A}, 1 \mathrm{~Hz})$ to evoke antidromic field potentials within the facial motor nucleus. These field potentials, recorded via the vector-filled pipette, were used to map the facial motor nucleus (FN) and identify the location of the VNC, which resides dorsal to the facial motor nucleus. Three $140 \mathrm{~nL}$ injections were performed $3000 \mu \mathrm{m}$ above the base of the medulla oblongata (determined as the lower limit of the facial field potential). The three injections were separated by $200 \mu \mathrm{m}$ and were aligned rostrocaudally. Successively, an optical fiber $(250 \mu \mathrm{m}$ core after desheathing; Thorlabs) was inserted $300 \mu \mathrm{m}$ above the injection site and secured to the skull through a cyanoacrylate adhesive. Prior to the implantation, the optical fibers were glued to a zirconia ferrule (outside diameter $1.25 \mathrm{~mm}$; bore $230 \mu \mathrm{m}$; Thorlabs).

\section{Injection of the fluoro-gold into the VNC or rostral ventrolateral medulla (RVLM)}

To examine the projection from the vestibular ganglion to the VNC, neuronal retrograde tracer (1\% Fluoro-Gold, Fluorochrome, LLC, CO) was injected into the left VNC using the above mentioned method. After the experiment, decalcifying liquid (Kalkitox, Wako, Tokyo, Japan) was applied to the left labyrinths. The same tracer was injected into the RVLM to examine the projection from the VNC to RVLM. For unilateral administration of vector in the RVLM, three $14 \mathrm{~nL}$ injections were made $300 \mu \mathrm{m}$ above the base of the medulla oblongata (determined as the lower limit of the facial field potential) and at the medial edge of the respiratory column (identified by respiratory synchronous multiunit activity). The three injections were separated by $200 \mu \mathrm{m}$ and were aligned rostrocaudally.

\section{Balance test using photostimulation}

A blue laser (473 nm; MBL-III-473, Changchun New Industries Optoelectronics Technology Co. Ltd.) and a green laser (532 nm; MBL-III-532, Changchun New Industries Optoelectronics Technology Co. Ltd.) were used to stimulate the ChR2 and eArchT of the neurons located in the VNC, respectively. The light output of each optical fiber was measured with a light meter (PM20A, Thorlabs), and the laser setting required to deliver $10 \mathrm{~mW}$ was recorded. Rats were briefly anesthetized with isoflurane while the connection between the implanted fiber optic and the laser delivery system was established. After recovery from the isoflurane inhalation, a rat was placed on a rod located $50 \mathrm{~cm}$ above the ground. The parameters related to photostimulation are as follows: duration, $10 \mathrm{~ms}$; frequency, $10,20,30$, or $40 \mathrm{~Hz}$. Photostimulation was also applied for a duration of $1 \mathrm{~s}$ (hold). Body tilt was recorded during photostimulation using a video camera (EX-100F, CASIO), and its degree was calculated using software (https://www.kinovea.org/).

\section{Measurement of arterial pressure (AP) and renal sympathetic nerve activity (RSNA)}

A polyethylene catheter (PE-50; Becton-Dickinson, Sparks, MD) was inserted into the abdominal aorta via the left femoral artery to measure AP. The catheter was exteriorized from the back of the neck. For recording the RSNA, the postganglionic renal sympathetic nerve was isolated through a right or left flank incision, and two stainless-steel electrodes (AS633; Cooner Wire, Chatworth, CA) were placed around it. The nerve and electrodes were covered and fixed with Low Toxicity Silicone Adhesive (Kwik-Sil, World Precision Instruments), and the electrodes were exteriorized at the back of the neck. The catheter was connected to a pressure transducer (MP5200; Baxter, Deerfield, IL) placed at the cardiac level. The signal from the transducer was transmitted to an amplifier (MEG-6108; Nihon Kohden, Tokyo, Japan). The electrodes for recording RSNA were connected to an amplifier (MEG-1200; Nihon Kohden, Tokyo, Japan) equipped with a 50- to $1000-\mathrm{Hz}$ band-pass filter. The output from the amplifier was passed through a gate circuit to subtract baseline noise, and it was rectified by an absolute value circuit. All signals were recorded using an analog-to-digital converter (PowerLab; AD Instruments, New South Wales, Australia) at a rate of $1000 \mathrm{~Hz}$.

\section{Immunohistochemistry}

Rats were euthanized with an overdose of pentobarbital sodium and perfused transcardially with $100 \mathrm{~mL}$ of heparinized saline, followed by $150 \mathrm{~mL}$ of freshly prepared $4 \%$ paraformaldehyde in sodium phosphate buffer ( $\mathrm{pH}$ 7.4). Subsequently, their brains were extracted and post-fixed at $4{ }^{\circ} \mathrm{C}$ for $24-48 \mathrm{~h}$ in the same fixative. Transverse sections ( $40 \mu \mathrm{m}$ thick) were then cut using a cryotome and stored in a cryoprotectant solution (20\% glycerol plus $30 \%$ ethylene glycol in $50 \mathrm{mM}$ phosphate buffer, $\mathrm{pH} 7.4$ ) at $-20{ }^{\circ} \mathrm{C}$. To confirm the expression of either the mCherry or eYFP in the VNC, the following antibodies were used: the mCherry protein was detected with the anti-DsRed (mouse monoclonal, 1:500; sc-390909 AF594; Santa Cruz Biotechnology); the eYFP protein was detected with the anti-GFP (chicken polyclonal, 1:500; GFP-1010; Aves Labs) followed by the Alexa Fluor-488-tagged rabbit anti-chicken antibody (1:200; Jackson ImmunoResearch Laboratories). To examine the $\mathrm{c}$-fos expression, the anti-c-fos antibody (1:1000; Millipore \#ABE457; EMD Millipore) was used, followed by the Alexa Fluor-594-tagged donkey anti-rabbit 
antibody (1:200; Jackson ImmunoResearch Laboratories). For the detection of Tyrosine Hydroxylase (TH)-expressing neurons, the anti-TH antibody (1:1000; Millipore \#AB1542; EMD Millipore) was used, followed by the Alexa Fluor-488-tagged donkey anti-sheep antibody (1:200; Jackson ImmunoResearch Laboratories). Thereafter, the brain sections were analyzed using fluorescence microscopy (BZ-X800, KEYENCE). The output levels were adjusted to include all the information-containing pixels, while the balance and contrast were adjusted to reflect the true rendering as much as possible. No other image retouching was performed.

\section{Vestibular lesion}

Sodium arsanilate solution $(100 \mathrm{mg} / \mathrm{mL})$ was injected into the bilateral middle-ear cavity ( $50 \mu \mathrm{L} / \mathrm{ear}$ ) for vestibular lesion (VL) under light sedation with isoflurane (Escain, Pfizer). Same volume of the saline was used for the sham operation (Sham). The success of VL was confirmed by observing the swimming behavior of the rats after they were gently placed in a small tub of water. Rats with complete VL were unable to determine the direction in which they had to swim to reach the surface and continued to turn around under water.

\section{Exposure to the hypergravity environment}

The hypergravity environment was induced by centrifugation using a custom-made gondola-type rotating box (Shimadzu). Rats were fed ad libitum, the cages were maintained on a 12:12-h light-dark cycle, and the room temperature was maintained at $24 \pm 1^{\circ} \mathrm{C}$. Rats were maintained in groups for the detection of c-fos in $\mathrm{C} 1$ neurons; however, rats were individually maintained in the case of the AP and RSNA measurements to avoid damage to the catheter and electrodes.

\section{Statistical analysis}

All the data sets were tested for normality using either the D'Agostino-Pearson omnibus normality or Kolmogorov-Smirnov tests. Equal variances were examined successively through the Brown-Forsythe test. If the criteria of normality and equal variance were satisfied, the statistical significance was evaluated using either one- or two-way ANOVA, followed by the Tukey-Kramer, Dunnett or Bonferroni tests. All values were expressed as mean $\pm \mathrm{SEM}$, while the statistical significance was set at $P<0.05$.

\section{Results}

\section{CAMK2-expressing neurons in the VNC are involved in balance function}

To measure the VNC receiving the gravitational inputs through the vestibular nerve, we injected the neuronal retrograde tracer (1\% fluoro-gold) into the VNC. Expression of fluoro-gold was observed in the ipsilateral vestibular ganglion 2 days after injection (Fig. 1b). Next, we examined the role of CAMK2-expressing neurons on the balance function. Opsin, ChR, or eArchT was expressed in CAMK2-expressing neurons in the unilateral VNC. We applied photostimulation to these neurons using a blue $(473 \mathrm{~nm})$ or green laser $(532 \mathrm{~nm})$ in the rat which was placed on the rod. Excitation (blue laser) of the CAMK2-expressing neurons in the VNC induced body tilt to the contralateral side, while the opposite response was observed by neural inhibition (green laser) (Fig. 2a and Video S1). In both cases, a significant increase in tilt angle was observed although the direction of the body tilt was different (Fig. 2b, c). This tilt angle was dependent on the intensity (stimulus frequency) of the photostimulation (Fig. 2d, e). Since we confirmed both the appropriate expression of the opsins, including both ChR2 and eArchT, and the placement of the fiber optics (Fig. 3a, b), it can be concluded that CAMK2-expressing neurons in the VNC participate in the balance function.

\section{CAMK2-expressing neurons in the VNC are involved in the autonomic response}

We investigated whether the CAMK2-expressing neurons in the VNC can induce a sympathetic and a cardiovascular response in rats. In the excitation of CAMK2-expressing neurons in the VNC, photostimulation for $10 \mathrm{~ms}$ showed an increase in the AP (Fig. 4a). This response was greater upon photostimulation for $1 \mathrm{~s}$, and the RSNA became prominent (Fig. 4b). On the other hand, inhibition for $10 \mathrm{~ms}$ of the CAMK2-expressing neurons in the VNC did not show any response; however, stronger photostimulation (1 s) showed an increase in both AP and RSNA (Fig. 4c, d). In these responses, both excitation and inhibition of CAMK2-expressing neurons in the VNC showed a photostimulation intensity-dependent (stimulus frequencydependent) increase in responses (Fig. 4e, g). With regard to the heart rate (HR) response, stronger photostimulation induced bradycardia (Fig. 4f). Interestingly, the photostimulation-induced $(1 \mathrm{~s})$ responses including AP, HR, and RSNA were not observed in the anesthetized rats (Fig. 5a-d), suggesting that autonomic responses induced by vestibular stimulation require a conscious condition. 
Fig. 2 a Photostimulation of either the ChR2 (blue) or the eArchT (green) in the CAMK2expressing neurons located in the vestibular nucleus complex (VNC) induced body tilt. Excitatory photostimulation induced body tilt to the contralateral side while inhibitory photostimulation showed the opposite response. Please see the Video S1. b, c Summarized data of the changes in body tilt angle induced by excitatory (b) or inhibitory (c) photostimulation. Statistics: unpaired $t$ test; $t(14)=6.726, P<0.0001$ (b) and $t(14)=3.446, P=0.0039$

(c). d, e Summarized data of the body tilt angle induced by changes in the frequency of excitatory (d) or inhibitory (e) photostimulation. A two-way ANOVA with Tukey's multiple comparisons test was applied; $F(4,56)=27.11, P<0.0001($ d) and $F(4,56)=29.96, P<0.0001$ (e). For all statistical analyses, single, double, or triple significance symbols indicate $P<0.05, P<0.01$, or $P<0.001$, respectively (color figure online)
A
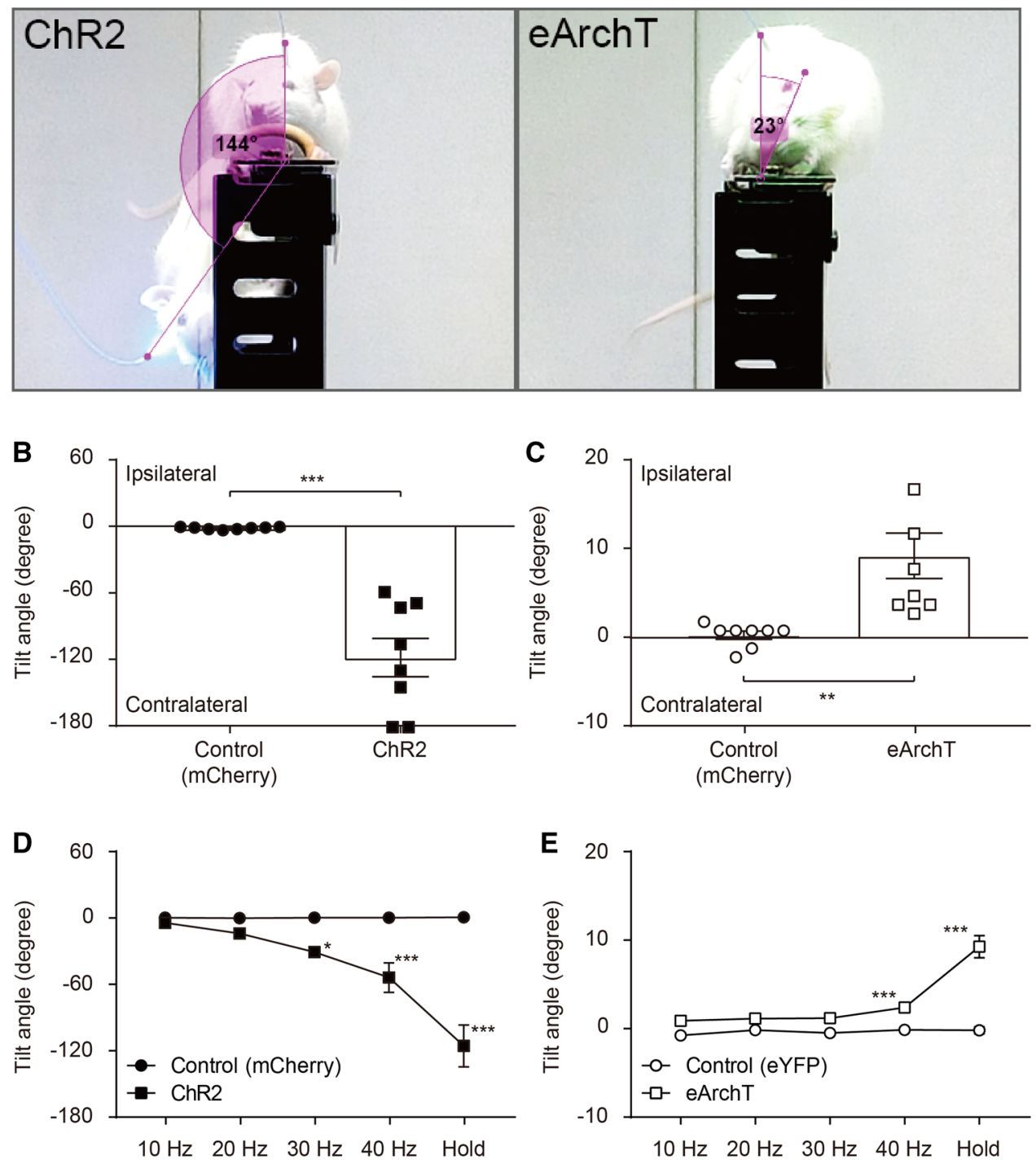

The present study demonstrated that not only the excitation but also the inhibition of the CAMK2-expressing neurons in the VNC resulted in an increase in RSNA. To investigate further, we measured the evoked potential of the renal sympathetic nerve induced by photostimulation of CAMK2-expressing neurons in the VNC in conscious rats. The nerve activity evoked by low-frequency photostimulation $(0.5 \mathrm{~Hz})$ was determined by constructing peristimulus waveform averages of the half-wave rectified signal (100 sweeps) (Fig. 6a-c). Excitatory photostimulation for a duration of $10 \mathrm{~ms}$ resulted in an evoked potential with a sharp peak (Fig. 6d); the onset latency was $75.1 \pm 1.7 \mathrm{~ms}(n=8)$. However, there was no evoked potential of the renal sympathetic nerve upon inhibitory photostimulation (Fig. 6d). Even upon inhibitory photostimulation, the evoked potential, which had a wide skirt form, was observed when the duration of the stimulation was set to $500 \mathrm{~ms}$ (Fig. 6d).

\section{Neural projection from the VNC to the RVLM}

To examine the neural circuit of the vestibulo-sympathetic reflex, we injected neuronal retrograde tracer (1\% fluorogold) into the RVLM (Fig. 7a). Bilateral neurons in the VNC were labeled, suggesting that the neurons in the VNC project into the RVLM bilaterally. Furthermore, the terminal end of the neurons in the VNC was observed in the RVLM; some terminals were observed alongside the $\mathrm{C} 1$ neurons (Fig. 7b). Next, we examined whether the vestibular stimulation activates $\mathrm{C} 1$ neurons in the RVLM. Exposure to a hypergravity environment for $90 \mathrm{~min}$ showed expression of c-fos in $\mathrm{C} 1$ neurons in the RVLM; this was significantly suppressed by VL (Fig. 7c, d). In non-C1 neurons in the 
Fig. 3 a Representative image of either ChR2 or eArchT expression in the CAMK2expressing neurons in the vestibular nucleus complex (VNC). The arrow indicates the trace of the fiber optic. The scale bar is $100 \mu \mathrm{m}$. b Location of the fiber optic tips for the excitatory (ChR2) or inhibitory (eArchT) photostimulation in the CAMK2-expressing neurons in the VNC. Distance from caudal to bregma $(\mathrm{mm})$ is shown at lower of each section

A
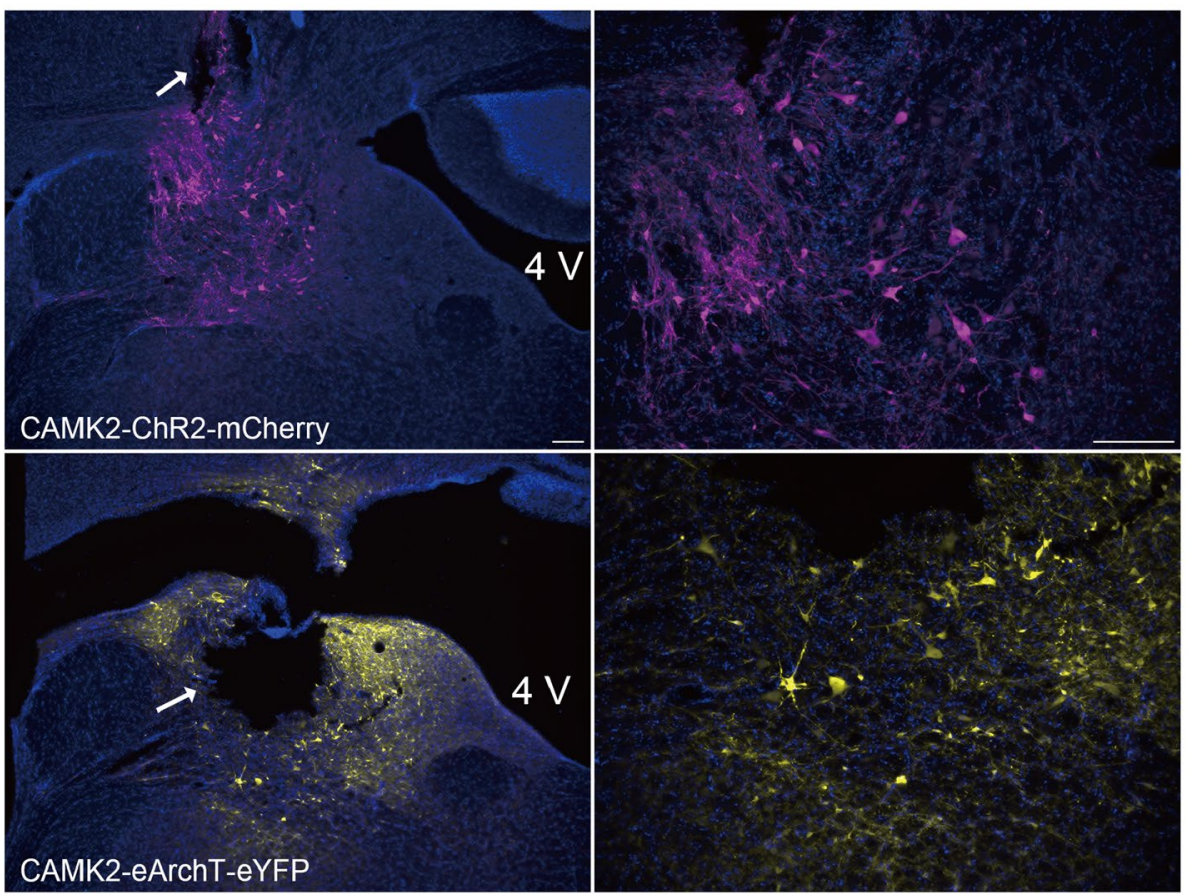

B

CAMK2-ChR2-mCherry $\square$ CAMK2-eArchT-eYFP

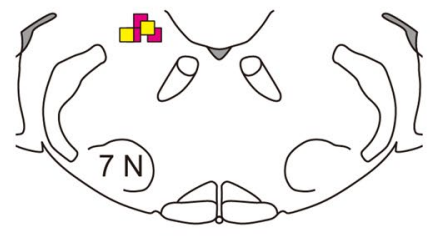

$-10.68 \mathrm{~mm}$

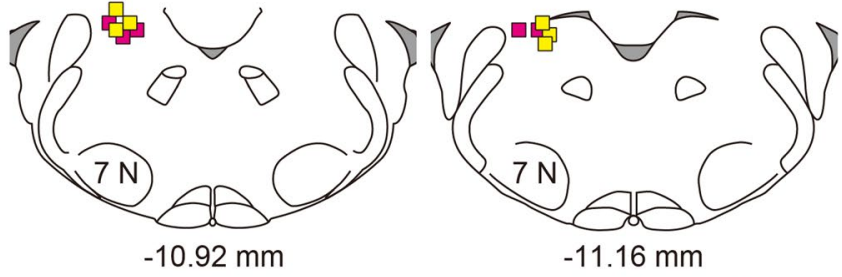

Bregma
RVLM, c-fos expression was also significantly suppressed by VL (Fig. 7d).

To examine whether the vestibulo-sympathetic response occurs due to the monosynaptic neural projection from the VNC to the RVLM, we measured sympathetic and cardiovascular responses during excitatory photostimulation of the terminal end of CAMK2-expressing neurons in the VNC which project into the RVLM. An increase in RSNA followed by the pressor response was observed upon photostimulation (1 s) (Fig. 8a). The photostimulation of either the right or left RVLM significantly increased RSNA and AP while also decreasing the HR (Fig. 8b-d). The onset latency of the evoked potential, measured using the same methods as those used to capture the data of Fig. 6, was $53 \pm 1.8 \mathrm{~ms}$. Thus, it is possible that the monosynaptic neural projection from the VNC to the RVLM is involved in the vestibulosympathetic reflex (Tables 1,2).

\section{Decrease in responsiveness of the neurons in the VNC induced by hypergravity exposure}

We have demonstrated that the sensitivity of the vestibulosympathetic reflex is attenuated by exposure to a hypergravity environment $[5,19]$. We examined whether the change in responsiveness of CAMK2-expressing neurons in the VNC induced by hypergravity exposure is involved in the attenuation of the vestibulo-sympathetic reflex. The rats which were implanted with an arterial catheter and electrodes for the recording of RSNA were maintained in the hypergravity environment for 5 days. The sympathetic and cardiovascular responses induced by photostimulation were significantly suppressed even on the first day of the hypergravity loading (Fig. 9a-d). The AP response was significantly decreased dependent on the exposed day (Fig. 9b). On the other hand, the responses of the HR and RSNA were decreased by the 3 rd day of the loading, and there was no difference in values between the 3rd and 5th day (Fig. 9c, d). This study demonstrated that the attenuation of the vestibulo-sympathetic reflex 
A

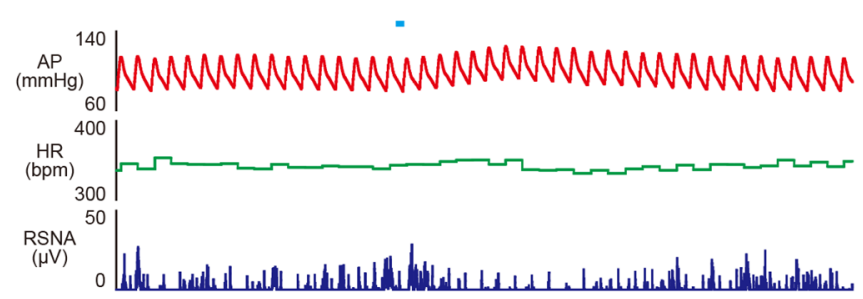

C

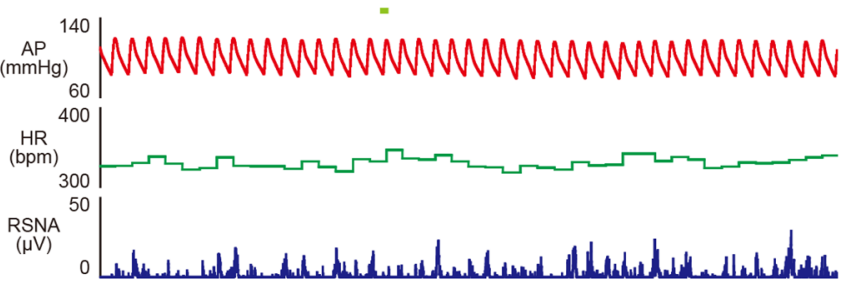

B

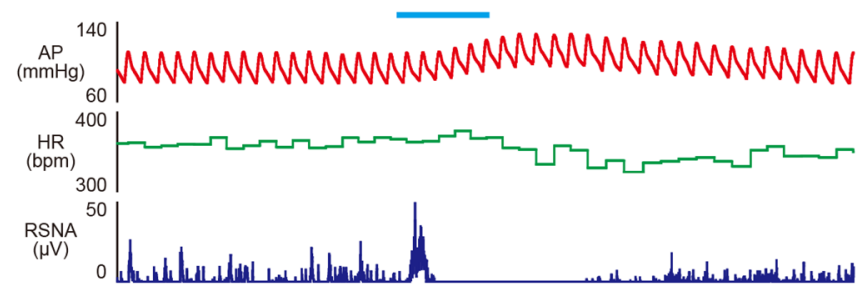

E

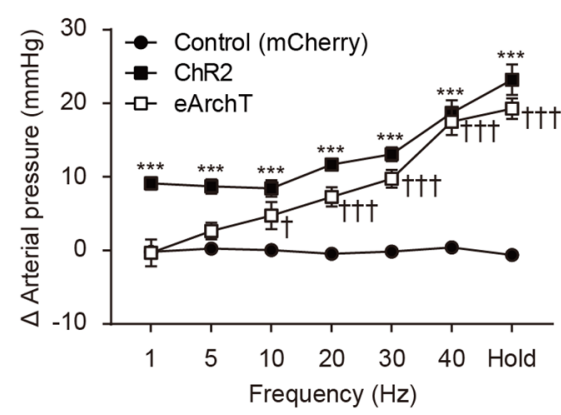

$\mathbf{F}$

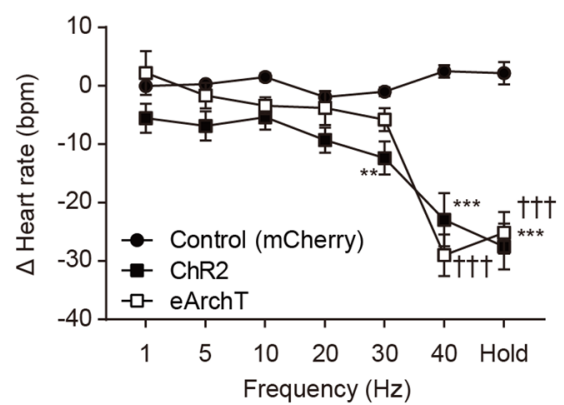

D

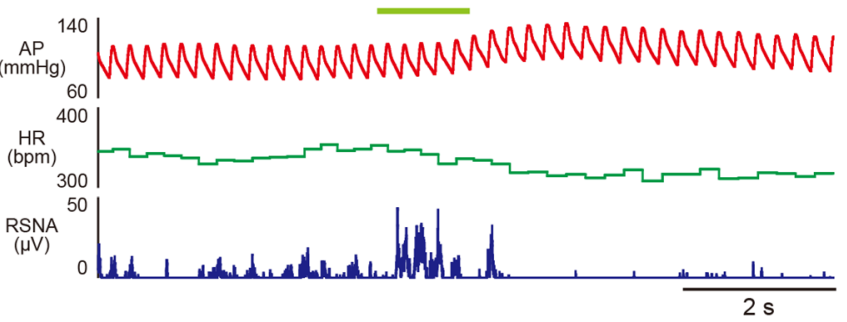

Fig. 4 a, b Effect of excitatory (a, b) or inhibitory (c, d) photostimulation of the CAMK2-expressing neurons in the vestibular nucleus complex (VNC) on the arterial pressure (AP), heart rate (HR), and renal sympathetic nerve activity (RSNA) in conscious rats. The duration was $10 \mathrm{~ms}(\mathbf{a}, \mathbf{c})$ or $1 \mathrm{~s}(\mathbf{b}, \mathbf{d})$. e-g Summarized data of change in AP (e), HR (f), and RSNA (g) induced by excitatory (ChR2) or inhibitory (eArchT) photostimulation of the CAMK2-expressing neurons in the VNC in conscious rats. For their control, the blue laser $(473 \mathrm{~nm})$ was applied to the rats into which the control vector (AAV-

induced by exposure to a hypergravity environment involves at least a decrease in responsiveness of CAMK2-expressing neurons in the VNC.

\section{Discussion}

The major findings of the present study are as follows: (1) CAMK2-expressing neurons in the $\mathrm{VNC}$ are involved in the balance function; (2) both excitation and inhibition of the CAMK2-expressing neurons in the $\mathrm{VNC}$ resulted in an increase in RSNA followed by the pressor response;
CAMK2-mCherry) was injected. The frequency of the photostimulation was varied from 1 to $40 \mathrm{~Hz}$. In this case, the duration of each pulse was $10 \mathrm{~ms}$. The frequency "Hold" means the photostimulation with the duration of $1 \mathrm{~s}$. A two-way ANOVA with Tukey's multiple comparisons test was applied; $F(12,126)=15.71, P<0.0001$ (e), $F(12,126)=10.66, P<0.0001$ (f) and $F(12,126)=4.174, P<0.0001$ (g). $*$ or ${ }^{\dagger}$ vs. Control (mCherry). For all statistical analyses, single or triple significance symbols indicate $P<0.05$ or $P<0.001$, respectively

(3) sympathetic and cardiovascular responses induced by the photostimulation involve the monosynaptic neural projection from the VNC to the RVLM; and (4) exposure to hypergravity environment-induced attenuation of the vestibulo-sympathetic reflex is due at least in part to a decrease in responsiveness of CAMK2-expressing neurons in the VNC. 
A
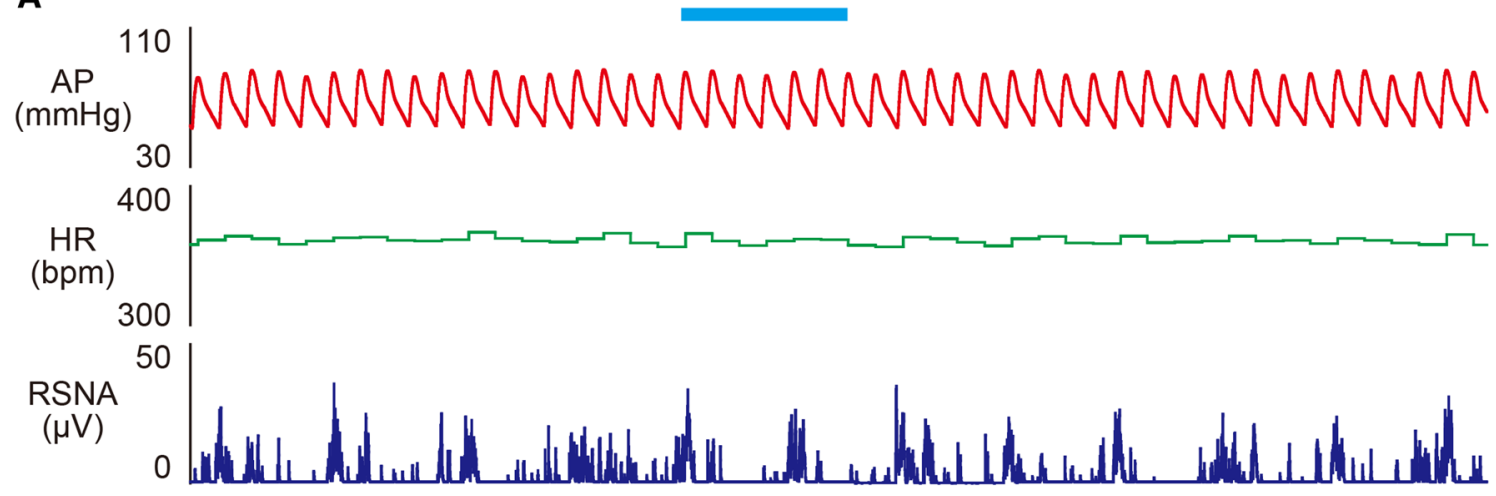

$2 \mathrm{~s}$

B

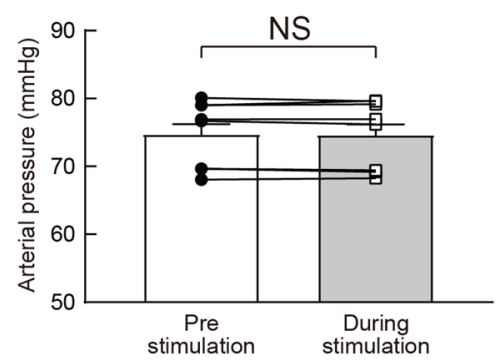

C

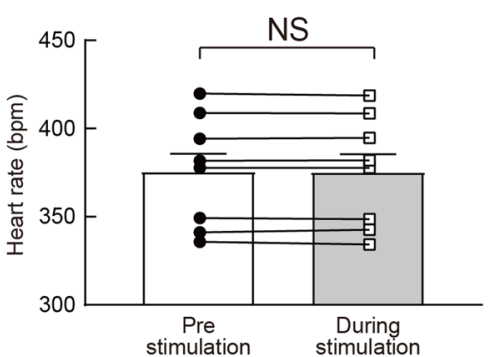

D

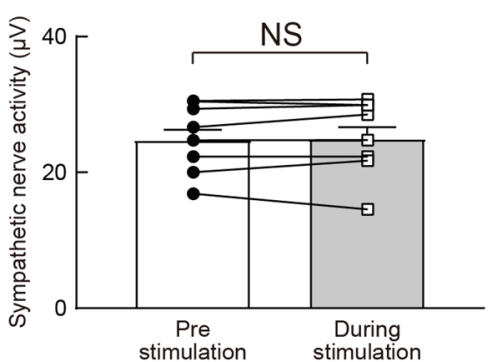

Fig. 5 a Effect of excitatory photostimulation of CAMK2-expressing neurons in the vestibular nucleus complex (VNC) on the arterial pressure (AP), heart rate (HR), and renal sympathetic nerve activity (RSNA) in an anesthetized rat. b-d Summarized data of the change

\section{Vestibulo-spinal reflex through the CAMK2-expressing neurons in the VNC}

Together with the cerebellum, the vestibular system contributes substantially to the stabilization of body posture during locomotion. In the present study, excitatory and inhibitory photostimulation of CAMK2-expressing neurons in the VNC resulted in body tilt, suggesting that these neurons are involved in the vestibulo-spinal reflex. The electrical stimulation of either the saccule or utricle induces the excitation of the ipsilateral vestibulo-spinal neurons [24, 25]. The vestibulo-spinal tract emerges from the VNC, especially the lateral vestibular nucleus, in the brainstem and runs unilaterally to the spinal motoneurons of the extensor muscles [26]. The present study showed that excitatory photostimulation induced body tilt to the contralateral side, suggesting that this might be a result of the contraction of the ipsilateral extensor muscles. Furthermore, the ablation of the neurons in the lateral vestibular nucleus using local injection of the diphtheria toxin resulted in both a change to the center of balance to the ipsilateral side and lower activation of the ipsilateral extensor muscle [26]. This is consistent with the data demonstrating that the inhibitory photostimulation in AP (b), HR (c), and RSNA (d) induced by excitatory (ChR2) photostimulation of CAMK2-expressing neurons in the VNC in anesthetized rats. The duration of the photostimulation was $1 \mathrm{~s}$

induced body tilt to the ipsilateral side. With regard to the subsets of the neurons in the $\mathrm{VNC}$, this area contains at least both glutamatergic and GABAergic/glycinergic neurons [27]. Selective expression of a reporter in excitatory neurons was observed using a $1.3 \mathrm{~kb}$ mouse camkII promoter in lentivirus pseudotyped with vesicular stomatitis virus glycoprotein [28]. However, the camkII promoter consistently drove gene expression in both inhibitory and excitatory neurons using adeno-associated virus (AAV) [29]. In the present study, we used AAV instead of a lentiviral vector, suggesting that opsins including ChR2 and eArchT were expressed in both the excitatory and inhibitory neurons in the VNC. It is suggested that the excitatory photostimulation of the GABAergic/glycinergic neurons in the VNC might inhibit the glutamatergic neurons in the contralateral VNC through the commissural inhibitory pathway [30], resulting in an emphasized body tilt to the ipsilateral side. 
Fig. 6 Representative data of excitatory (ChR2, a) or inhibitory (eArchT, b, c) photostimulation of the CAMK2-expressing neurons in the vestibular nucleus complex (VNC). Photostimulation produced a robust activation of renal sympathetic nerve activity (RSNA) in conscious rats. The frequency of the photostimulation is $0.5 \mathrm{~Hz}$. The duration of the photostimulation is $10 \mathrm{~ms}$ (a, b) or $500 \mathrm{~ms}$ (c). d Averaged data of the evoked potential in RSNA induced by excitatory or inhibitory photostimulation of the CAMK2-expressing neurons in the VNC. The data were superimposed 100 times

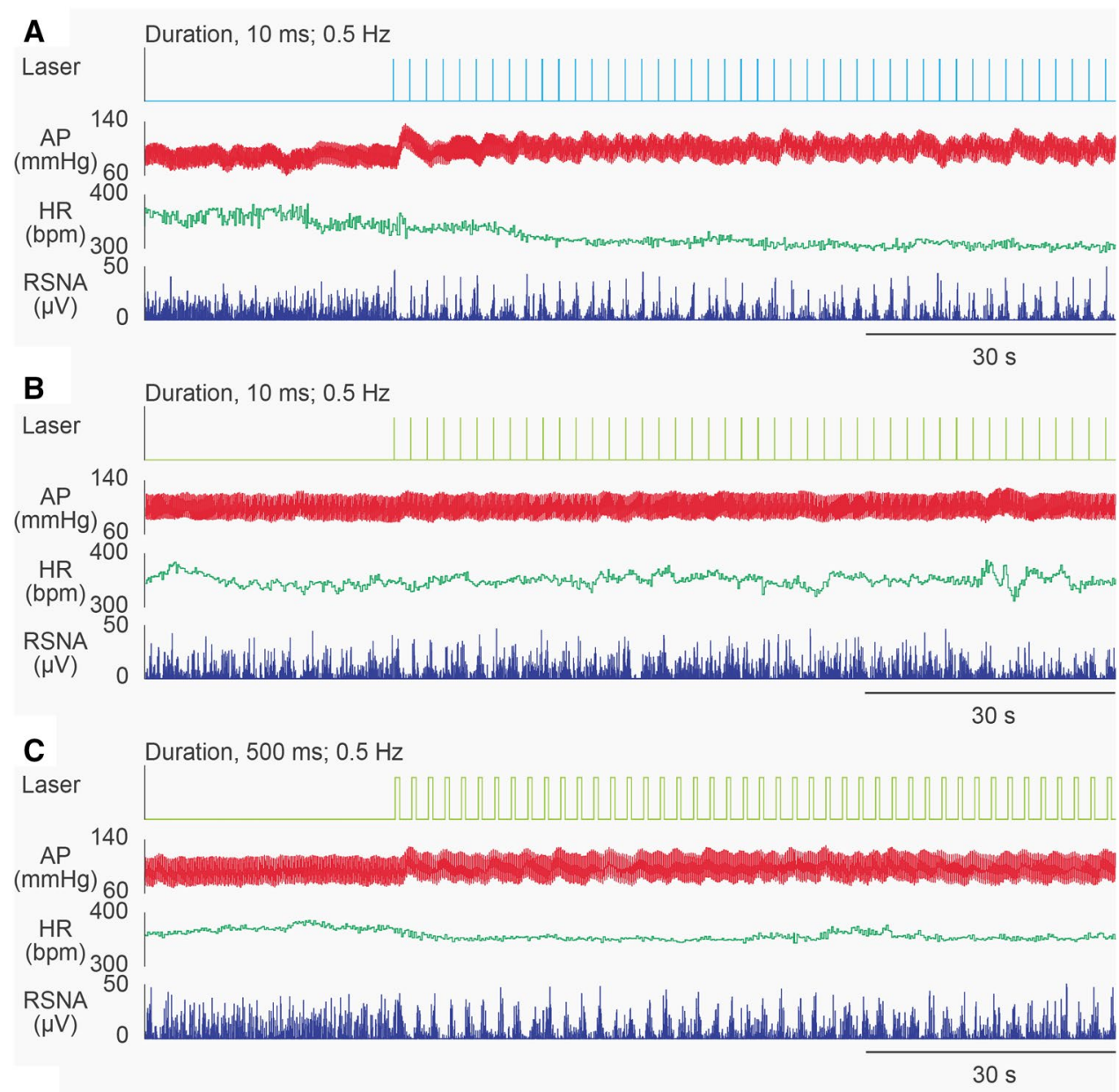

D

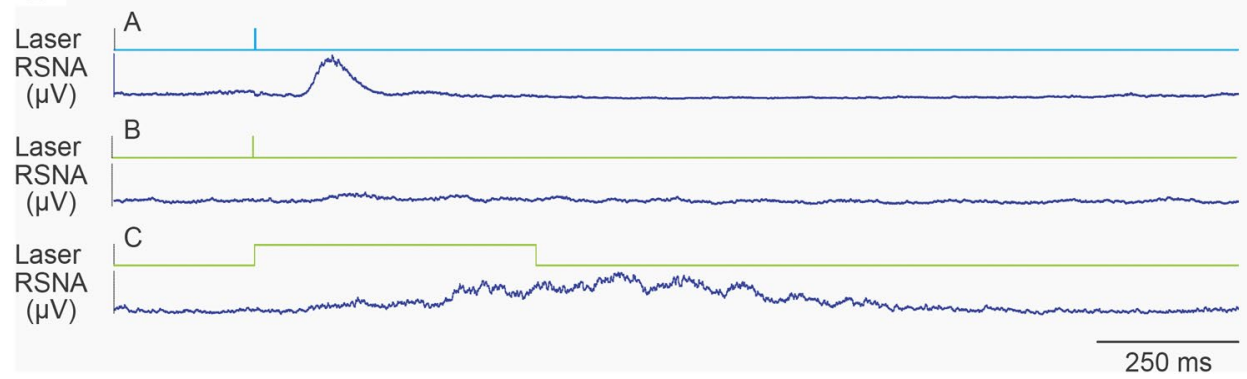

\section{Vestibulo-sympathetic reflex through the CAMK2-expressing neurons in the VNC}

The present study showed that both excitation and inhibition of the CAMK2-expressing neurons in the VNC induced sympathoexcitation followed by the pressor response. This is consistent with our previous data demonstrating that both exposure to microgravity and hypergravity induced the pressor response through the sympathetic nervous system [5, 31]. Furthermore, these responses were not observed in anesthetized rats [32]. This is in agreement with the data showing that the photostimulation of CAMK2-expressing neurons in the VNC induced neither sympathetic/cardiovascular nor body tilt, suggesting that the vestibulo-sympathetic reflex functions only in the conscious condition. In anesthetized cats, the firing rate of the VNC neurons was changed by the stimulation using wobble rotation [33]. Since the photostimulation of RVLM neurons including Phox2b- or Dopamine beta-hydroxylase-expressing neurons induced sympathoexcitation even in the anesthetized condition $[34,35]$, it is possible that presynaptic inhibition can be considered in the vestibulo-sympathetic reflex under anesthesia.

CAMK2-expressing neuron-induced sympathoexcitation might occur through stimulation of the excitatory neurons as it is reported that glutamatergic, rather than GABAergic neurons, in the VNC project to the RVLM 
Fig. 7 a Neuronal retrograde tracer (Fluoro-Gold) was injected at the left rostral ventrolateral medulla (RVLM). Fluoro-gold expression was observed in the vestibular nucleus complex (VNC). Scale bar: $200 \mu \mathrm{m}$ for main panels and $100 \mu \mathrm{m}$ for insets. b Axonal terminal of the neurons in the VNC at the RVLM. Viral vector, AAV-CAMK2-mCherry, was injected at the VNC. The scale bar is $100 \mu \mathrm{m}$. c c-fos expression in $\mathrm{C} 1$ neurons induced by exposure to hypergravity environment $(2 \mathrm{~g})$ for $90 \mathrm{~min}$ in rats with vestibular lesion (VL) and their sham operation (Sham). The scale bar is $100 \mu \mathrm{m}$. d Quantitative analysis of c-fos expression induced by $2 \mathrm{~g}$ loading in Sham and VL rats. Statistics: unpaired $t$ test; $t(14)=1.017, P=0.3266$ $[\mathrm{TH}(+) \operatorname{Fos}( \pm)], t(14)=10.26$, $P<0.0001[\mathrm{TH}(+)$ Fos $(+)]$, and $t(14)=5.713, P<0.0001$ [TH(-) Fos(+)]
A
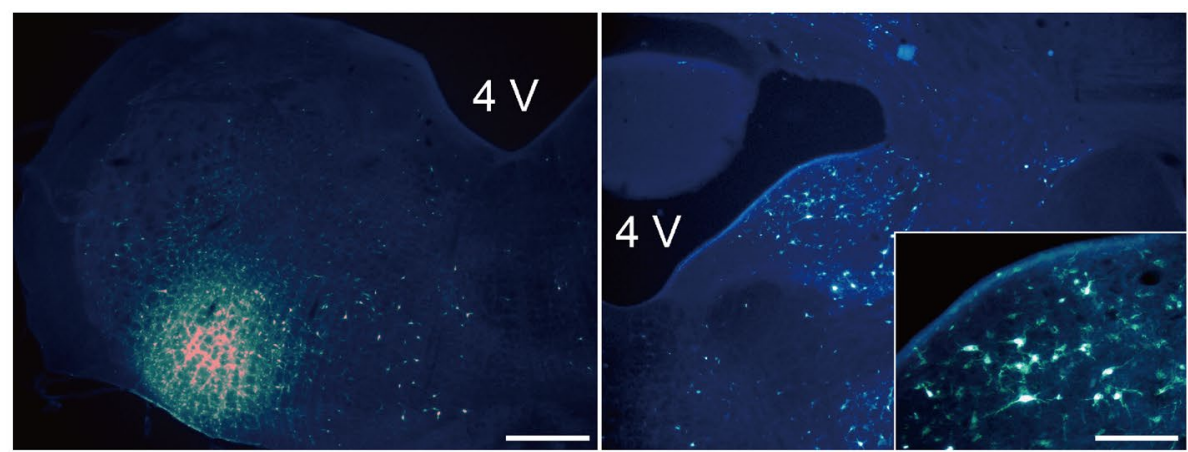

B

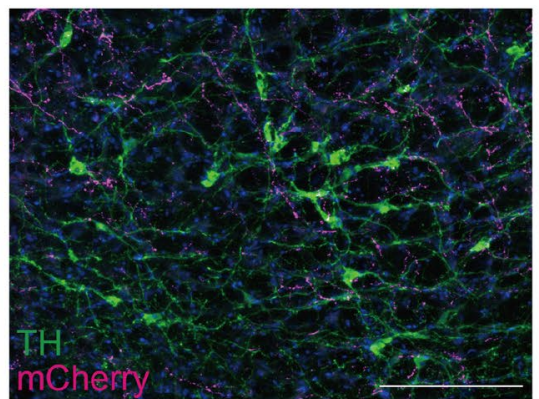

D

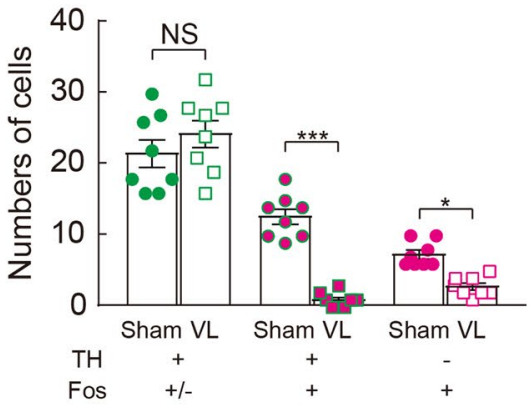

c Sham
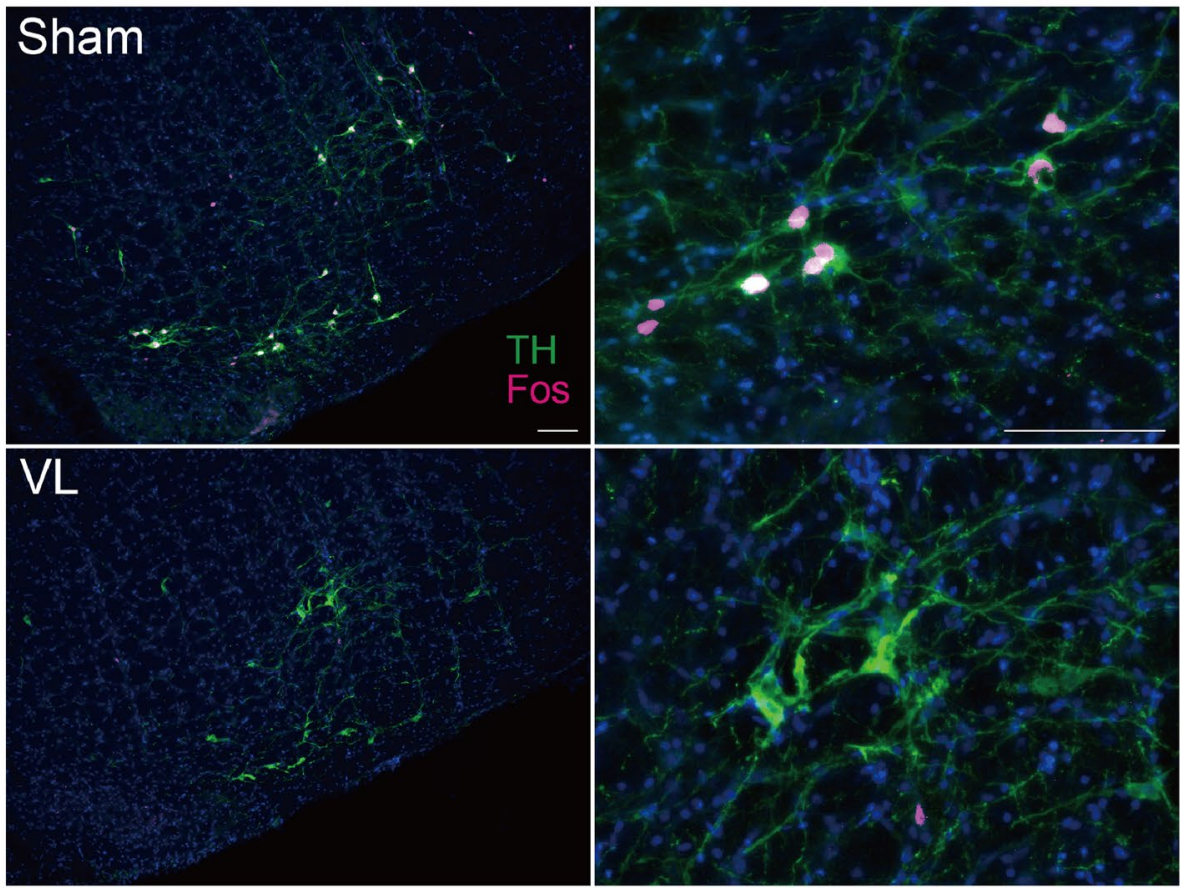

[27]. Even though the same response was observed here, the mechanism of excitation and inhibition of the CAMK2-expressing neuron-induced sympathoexcitation might be different because the form of the evoked potential in the renal sympathetic nerve was not the same (Fig. 6). In the case of excitatory photostimulation, activation of the monosynaptic neural projection from the VNC to the RVLM is involved in the sympathoexcitation because stimulation of the terminal end in the VNC neurons which project into the RVLM showed sympathoexcitation. Previous data are also supportive of this; onset latency was $40.8 \pm 0.9 \mathrm{~ms}$ in the stimulation of RVLM neurons [35], and a longer onset latency was observed in the photostimulation of neurons in the VNC $(75.1 \pm 1.7 \mathrm{~ms}$ in cell body stimulation and $53 \pm 1.8 \mathrm{~ms}$ in terminal end stimulation). However, the onset latency of the evoked potential in the renal sympathetic nerve was not the same in the inhibitory photostimulation of the CAMK2-expressing neurons in the 
A

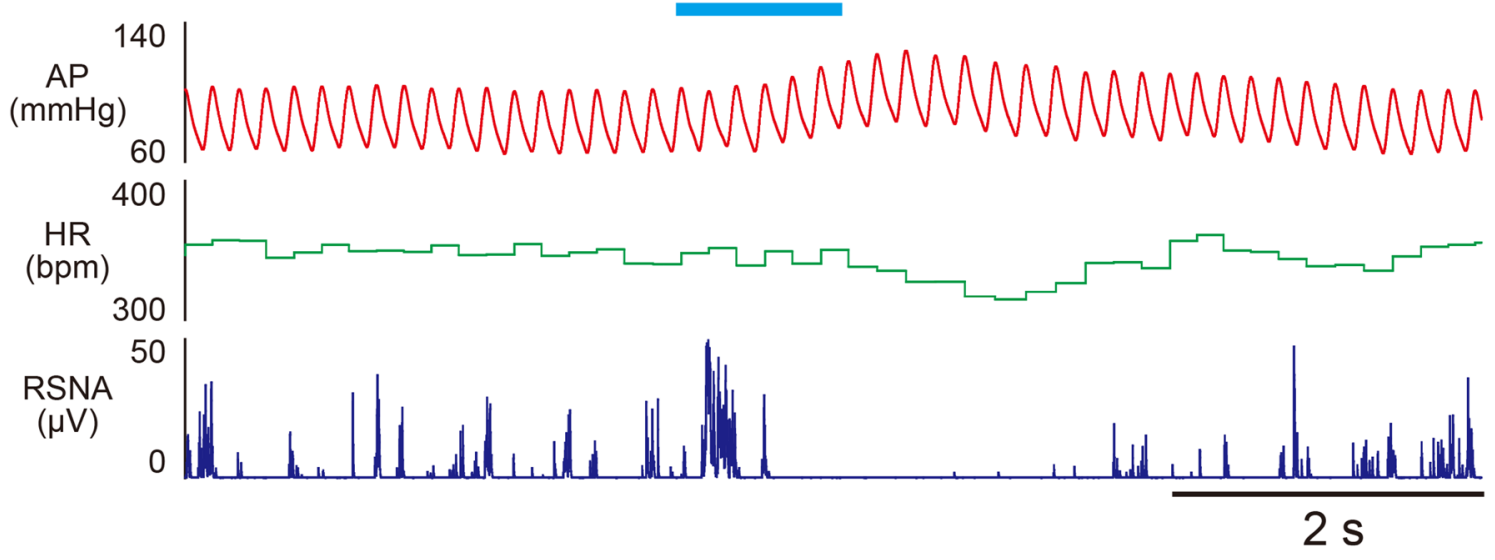

B

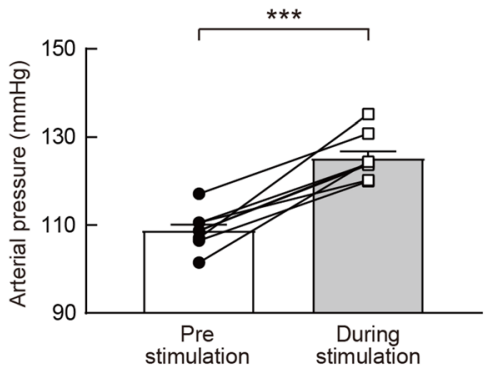

C

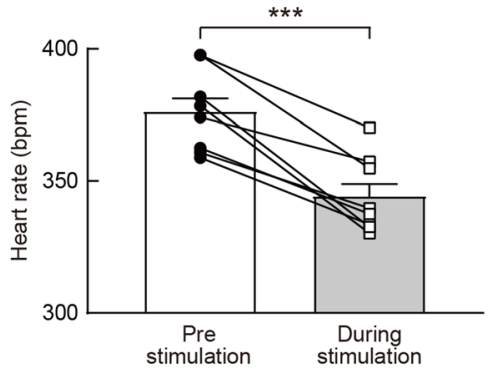

E

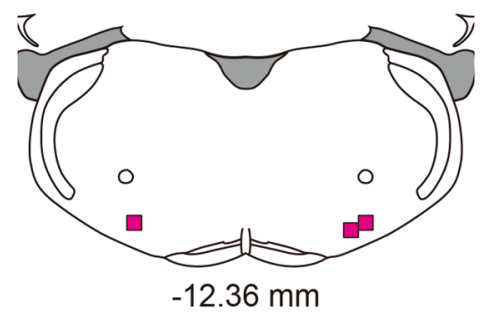

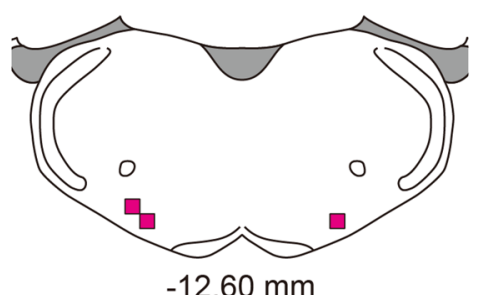

$-12.60 \mathrm{~mm}$

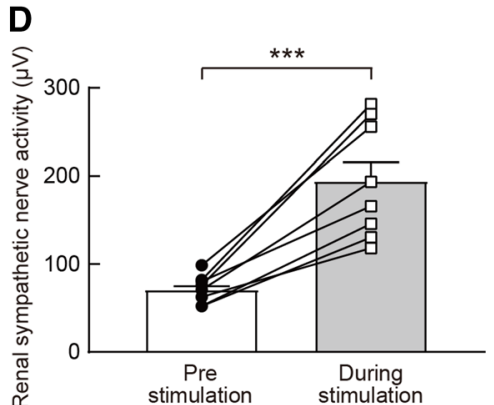

stimulation stimulation 
Table 1 Baseline data of arterial pressure and heart rate in Fig. 4

\begin{tabular}{|c|c|c|c|c|c|c|c|}
\hline & $1 \mathrm{~Hz}$ & $5 \mathrm{~Hz}$ & $10 \mathrm{~Hz}$ & $20 \mathrm{~Hz}$ & $30 \mathrm{~Hz}$ & $40 \mathrm{~Hz}$ & Hold \\
\hline \multicolumn{8}{|c|}{ Baseline data of arterial pressure } \\
\hline Control & $105.7 \pm 2.2$ & $106.5 \pm 1.9$ & $105.5 \pm 1.7$ & $106.7 \pm 1.3$ & $107.3 \pm 1$ & $107.9 \pm 0.7$ & $108.3 \pm 1$ \\
\hline ChR2 & $105.3 \pm 1.4$ & $105.5 \pm 1.6$ & $107.3 \pm 1.5$ & $106.6 \pm 1.6$ & $105.7 \pm 1.7$ & $105.8 \pm 1.4$ & $107 \pm 1.7$ \\
\hline eArchT & $105.1 \pm 2.9$ & $105.3 \pm 2.2$ & $106.2 \pm 2.3$ & $105.6 \pm 2.1$ & $105.9 \pm 2.3$ & $105.9 \pm 1.3$ & $105.8 \pm 1.6$ \\
\hline \multicolumn{8}{|c|}{ Baseline data of heart rate } \\
\hline Control & $347 \pm 13.8$ & $346.5 \pm 12.7$ & $344.7 \pm 13.2$ & $345.1 \pm 11.4$ & $346.3 \pm 11.1$ & $344.4 \pm 11.2$ & $345.1 \pm 11.7$ \\
\hline ChR2 & $345 \pm 6.5$ & $337.2 \pm 6.6$ & $350.1 \pm 6.7$ & $346.2 \pm 7.2$ & $351.6 \pm 8.6$ & $362.2 \pm 10.3$ & $361.1 \pm 11$ \\
\hline eArchT & $322 \pm 10.2$ & $324.8 \pm 12.6$ & $331 \pm 10.7$ & $330.8 \pm 8.9$ & $329.8 \pm 10.1$ & $371.2 \pm 10.4$ & $369.8 \pm 12.3$ \\
\hline
\end{tabular}

Table 2 Baseline data of arterial pressure and heart rate in Fig. 9

\begin{tabular}{lllll}
\hline & Pre-HG & HG-D1 & HG-D3 & HG-D5 \\
\hline Arterial pressure & $109.2 \pm 1.7$ & $111 \pm 1.1$ & $110.8 \pm 3.8$ & $100.9 \pm 2.5$ \\
Heart rate & $351.3 \pm 12.9$ & $328 \pm 3.2$ & $373.4 \pm 7$ & $392.9 \pm 6.9$ \\
\hline
\end{tabular}

of autonomic responses [38]. C1 neurons are differentially activated by many types of stressors including hypoglycemia, infection or inflammation, hypoxia, nociception, and hypotension. Since the gravitational change $(2 \mathrm{~g})$ can be considered as one of the stressors, $\mathrm{C} 1$ neurons might be part of the vestibulo-cardiovascular reflex. In the RVLM, the neurons in the VNC are primarily directed toward the somata and proximal dendrites of non-catecholaminergic neurons, with minor projections to the distal dendrites of catecholaminergic cells [39]. On the other hand, some neurons in the VNC project to the nucleus of the solitary tract [40], which could activate the neurons in the RVLM [38]. Accordingly, it is possible that $\mathrm{C} 1$ neuron activation by vestibular stimulation is a result of an indirect pathway.

Present study showed that CAMK2-expressing neurons in the VNC induced sympathoexcitation followed by the pressor response. However, increase in HR was not observed although sympathetic nerve activity was increased. Strong excitatory photostimulation $(30 \mathrm{~Hz}$, $40 \mathrm{~Hz}$, and hold) significantly decreased HR (Fig. 4f). This might be due to the activation of the baroreflex because of the following reasons: (1) HR response was observed after the pressor response, and (2) the inverse relationship was observed between AP and HR response. On the other hand, we have demonstrated that excitatory photostimulation of $\mathrm{C} 1$ neurons induces both sympathoexcitation and activation of the vagal efferent in mice [34]. Accordingly, it is possible that the photostimulation-induced no change in HR is due to the activation of both the sympathetic and the vagal efferent nerve.

\section{Plastic alteration of the vestibulo-sympathetic response through the CAMK2-expressing neurons in the VNC}

Previously, we reported that attenuation of the vestibulo-cardiovascular reflex occurred through exposure to a hypergravity environment. Specifically, the AP response induced by linear acceleration was suppressed in rats which were maintained in a $3 \mathrm{~g}$ environment for 6 days [21]. This was due to the decrease in phasic inputs of the peripheral vestibular organ because the rear-up behavior and head movement was significantly suppressed in the hypergravity environment $[20,21]$. The attenuation of the vestibulo-cardiovascular reflex was rescued by electrical vestibular stimulation during hypergravity loading [21]. The present study showed that the attenuation of the vestibulo-sympathetic reflex occurs after just 1 day of $2 \mathrm{~g}$ loading. Furthermore, this attenuation is due at least in part to a decrease in the responsiveness of CAMK2-expressing neurons in the VNC. The AP response induced by other stressors, with the exception of vestibular stimulation such as a loud noise and an air jet, was not changed by hypergravity loading [5], suggesting that the attenuation of the vestibulo-sympathetic reflex is due to the plastic alteration of the CAMK2-expressing neurons in the VNC rather than the neurons in the RVLM. Furthermore, this can explain that $2 \mathrm{~g}$ loading does not induce the saturation of the sympathetic nerve activity; i.e., there is a remaining capacity to induce sympathoexcitation. Since a change in gene expression occurred in the vestibular ganglion upon 2 g loading in rats [23], a decrease in responsiveness of CAMK2-expressing neurons in the VNC might be due to the influence of upstream signals. This should be examined in future studies.

\section{Conclusion}

In conclusion, we clarified that CAMK2-expressing neurons in the VNC participate in both balance function and the sympathetic/cardiovascular response. The monosynaptic 
A
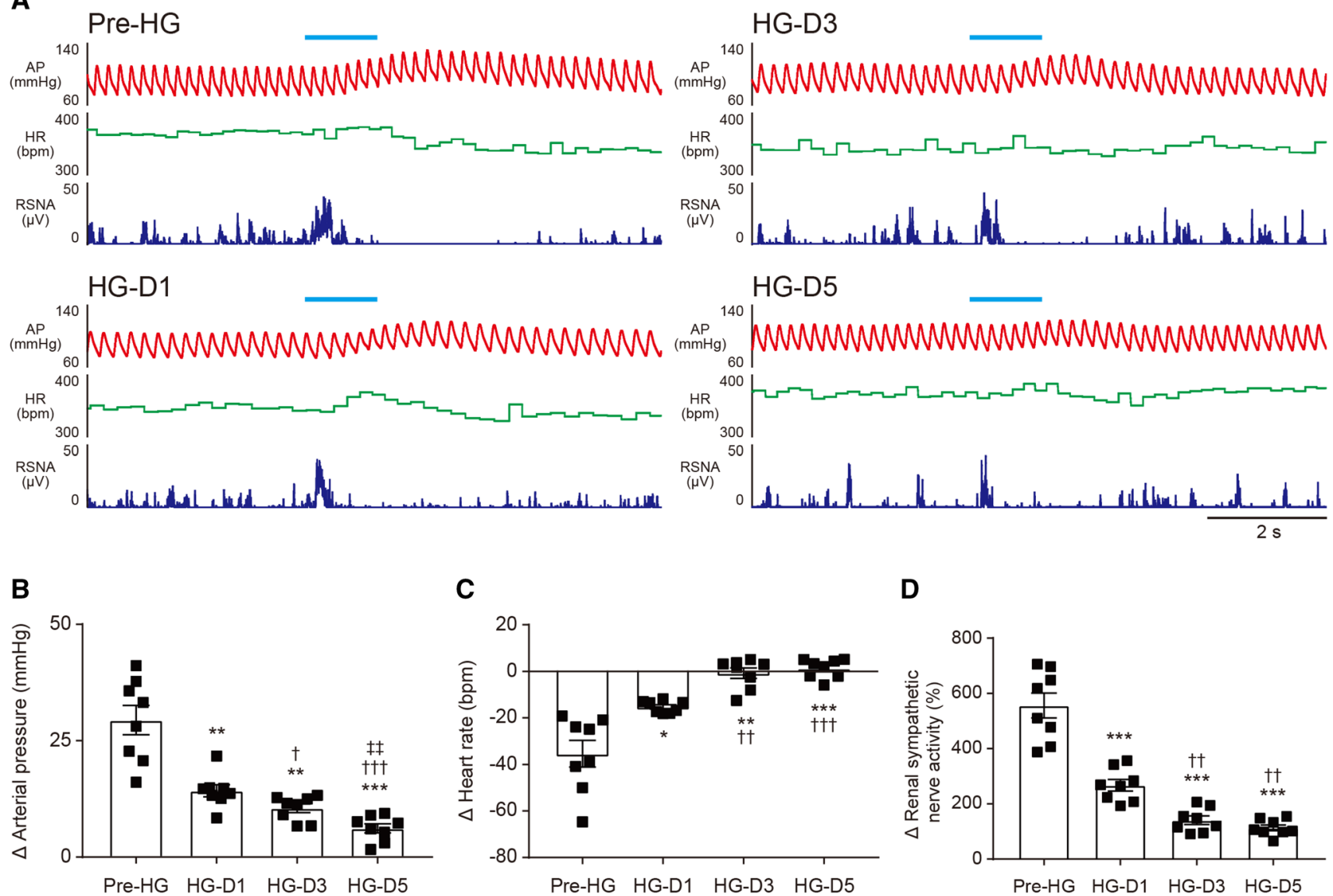

Fig. 9 a Effect of excitatory photostimulation of CAMK2-expressing neurons in the vestibular nucleus complex (VNC) on the arterial pressure (AP), heart rate (HR), and renal sympathetic nerve activity (RSNA) before and during (1st, 3rd, and 5th day) exposure to a hypergravity $(2 \mathrm{~g})$ environment in a conscious rat. The duration of the photostimulation is $1 \mathbf{s}$. b-d Summarized data of the change in AP (b), HR (c), and RSNA (d) induced by excitatory (ChR2) photostimulation of the CAMK2-expressing neurons in the VNC before

neural projection from the VNC to the RVLM is involved in the vestibulo-sympathetic pathway. Furthermore, the plasticity of the vestibulo-sympathetic response induced by gravitational change is due to a decrease in responsiveness of CAMK2-expressing neurons in the VNC. These neurons can be a target for vestibular training of elderly people and astronauts to prevent vestibular-related autonomic dysfunction.

Acknowledgements Special thanks to the Grant-in-Aid for Scientific Research on Innovative Areas 15H05940 (HM) and 18H04974 (CA), the Grant-in-Aid for Scientific Research (C) 18K06850 (HM) and 19K07283 (CA), The Takeda Science Foundation (CA), The Uehara Memorial Foundation (CA), The Kato Memorial Bioscience Foundation (CA), The Nakatomi Foundation (CA), The Mochida Memorial Foundation for Medical and Pharmaceutical Research (CA), The Daiichi Sankyo Foundation of Life Science (CA), The Inamori Grant (CA), Japan Aerospace Exploration Agency (HM) and Gifu University (CA). We also thank Editage (http://www.editage.jp) for English language editing. and during $2 \mathrm{~g}$ loading in conscious rats. The duration of the photostimulation is $1 \mathrm{~s}$. A two-way ANOVA with Tukey's multiple comparisons test was applied; $F(7,21)=50.8, P<0.0001$ (b), $F(7$, $21)=25.52, P=0.0006(\mathbf{c})$ and $F(7,21)=67.45, P<0.0001(\mathbf{d}) . *$ vs. Pre-HG, ${ }^{\dagger}$ vs. HG-D1, ${ }^{\ddagger}$ vs. HG-D3. For all statistical analyses, single, double, or triple significance symbols indicate $P<0.05, P<0.01$, or $P<0.001$, respectively

Author contribution CA and HM designed the study protocols; CA, YY, YM, TM, and HM conducted experiments and acquired and analyzed the data; and CA and HM wrote the manuscript.

\section{Compliance with ethical standards}

Conflict of interests The authors declare no competing interests.

Open Access This article is distributed under the terms of the Creative Commons Attribution 4.0 International License (http://creativeco mmons.org/licenses/by/4.0/), which permits unrestricted use, distribution, and reproduction in any medium, provided you give appropriate credit to the original author(s) and the source, provide a link to the Creative Commons license, and indicate if changes were made. 


\section{References}

1. Highstein SM, Holstein GR (2006) The anatomy of the vestibular nuclei. Prog Brain Res 151:157-203

2. Cullen KE (2012) The vestibular system: multimodal integration and encoding of self-motion for motor control. Trends Neurosci 35:185-196

3. Macefield VG, James C (2016) Superentrainment of muscle sympathetic nerve activity during sinusoidal galvanic vestibular stimulation. J Neurophysiol 116:2689-2694

4. Yates BJ, Bolton PS, Macefield VG (2014) Vestibulo-sympathetic responses. Compr Physiol 4:851-887

5. Abe C, Tanaka K, Awazu C, Chen H, Morita H (2007) Plastic alteration of vestibulo-cardiovascular reflex induced by 2 weeks of 3-G load in conscious rats. Exp Brain Res 181:639-646

6. Hammam E, Macefield VG (2017) Vestibular modulation of sympathetic nerve activity to muscle and skin in humans. Front Neurol $8: 334$

7. Tanaka K, Abe C, Awazu C, Morita H (2009) Vestibular system plays a significant role in arterial pressure control during head-up tilt in young subjects. Auton Neurosci Basic Clin 148:90-96

8. Abe C, Kawada T, Sugimachi M, Morita H (2011) Interaction between vestibulo-cardiovascular reflex and arterial baroreflex during postural change in rats. J Appl Physiol 111:1614-1621

9. Morita H, Abe C, Tanaka K (2016) Long-term exposure to microgravity impairs vestibulo-cardiovascular reflex. Sci Rep 6:33405

10. Kawao N, Morita H, Obata K, Tamura Y, Okumoto K, Kaji H (2016) The vestibular system is critical for the changes in muscle and bone induced by hypergravity in mice. Physiol Rep 4:e12979

11. Abe C, Tanaka K, Iwata C, Morita H (2010) Vestibular-mediated increase in central serotonin plays an important role in hypergravity-induced hypophagia in rats. J Appl Physiol 109:1635-1643

12. Murakami DM, Erkman L, Hermanson O, Rosenfeld MG, Fuller CA (2002) Evidence for vestibular regulation of autonomic functions in a mouse genetic model. Proc Natl Acad Sci USA 99:17078-17082

13. Fuller PM, Jones TA, Jones SM, Fuller CA (2002) Neurovestibular modulation of circadian and homeostatic regulation: vestibulohypothalamic connection? Proc Natl Acad Sci USA 99:15723-15728

14. Reschke MF, Bloomberg JJ, Harm DL, Paloski WH, Layne C, McDonald V (1998) Posture, locomotion, spatial orientation, and motion sickness as a function of space flight. Brain Res Brain Res Rev 28:102-117

15. Clarke AH, Grigull J, Mueller R, Scherer H (2000) The threedimensional vestibulo-ocular reflex during prolonged microgravity. Exp Brain Res 134:322-334

16. Reschke MF, Anderson DJ, Homick JL (1986) Vestibulo-spinal response modification as determined with the H-reflex during the Spacelab-1 flight. Exp Brain Res 64:367-379

17. Hallgren E, Migeotte PF, Kornilova L, Deliere Q, Fransen E, Glukhikh D, Moore ST, Clement G, Diedrich A, MacDougall H, Wuyts FL (2015) Dysfunctional vestibular system causes a blood pressure drop in astronauts returning from space. Sci Rep 5:17627

18. Abe C, Tanaka K, Awazu C, Morita H (2008) The vestibular system is integral in regulating plastic alterations in the pressor response to free drop mediated by the non-vestibular system. Neurosci Lett 445:149-152

19. Abe C, Tanaka K, Awazu C, Morita H (2008) Impairment of vestibular-mediated cardiovascular response and motor coordination in rats born and reared under hypergravity. Am J Physiol Regulat Integr Comp Physiol 295:R173-180

20. Abe C, Shibata A, Iwata C, Morita H (2010) Restriction of rearup-behavior-induced attenuation of vestibulo-cardiovascular reflex in rats. Neurosci Lett 484:1-5
21. Abe C, Tanaka K, Awazu C, Morita H (2009) Galvanic vestibular stimulation counteracts hypergravity-induced plastic alteration of vestibulo-cardiovascular reflex in rats. J Appl Physiol 107:1089-1094

22. Ross MD (1994) A spaceflight study of synaptic plasticity in adult rat vestibular maculas. Acta Otolaryngol Suppl 516:1-14

23. Uno Y, Horii A, Uno A, Fuse Y, Fukushima M, Doi K, Kubo T (2002) Quantitative changes in mRNA expression of glutamate receptors in the rat peripheral and central vestibular systems following hypergravity. J Neurochem 81:1308-1317

24. Kushiro K, Zakir M, Sato H, Ono S, Ogawa Y, Meng H, Zhang X, Uchino Y (2000) Saccular and utricular inputs to single vestibular neurons in cats. Exp Brain Res 131:406-415

25. Uchino Y, Sato H, Sasaki M, Imagawa M, Ikegami H, Isu N, Graf W (1997) Sacculocollic reflex arcs in cats. J Neurophysiol 77:3003-3012

26. Murray AJ, Croce K, Belton T, Akay T, Jessell TM (2018) Balance control mediated by vestibular circuits directing limb extension or antagonist muscle co-activation. Cell Rep 22:1325-1338

27. Holstein GR, Friedrich VL Jr, Martinelli GP (2016) Glutamate and GABA in vestibulo-sympathetic pathway neurons. Front Neuroanat 10:7

28. Dittgen T, Nimmerjahn A, Komai S, Licznerski P, Waters J, Margrie TW, Helmchen F, Denk W, Brecht M, Osten P (2004) Lentivirus-based genetic manipulations of cortical neurons and their optical and electrophysiological monitoring in vivo. Proc Natl Acad Sci USA 101:18206-18211

29. Nathanson JL, Yanagawa Y, Obata K, Callaway EM (2009) Preferential labeling of inhibitory and excitatory cortical neurons by endogenous tropism of adeno-associated virus and lentivirus vectors. Neuroscience 161:441-450

30. Uchino Y, Sato H, Zakir M, Kushiro K, Imagawa M, Ogawa Y, Ono S, Meng H, Zhang X, Katsuta M, Isu N, Wilson VJ (2001) Commissural effects in the otolith system. Exp Brain Res 136:421-430

31. Abe C, Tanaka K, Awazu C, Morita H (2008) Strong galvanic vestibular stimulation obscures arterial pressure response to gravitational change in conscious rats. J Appl Physiol 104:34-40

32. Gotoh TM, Fujiki N, Tanaka K, Matsuda T, Gao S, Morita H (2004) Acute hemodynamic responses in the head during microgravity induced by free drop in anesthetized rats. Am J Physiol Regulat Integr Comp Physiol 286:R1063-1068

33. McCall AA, Miller DM, DeMayo WM, Bourdages GH, Yates BJ (2016) Vestibular nucleus neurons respond to hindlimb movement in the conscious cat. J Neurophysiol 116:1785-1794

34. Abe C, Inoue T, Inglis MA, Viar KE, Huang L, Ye H, Rosin DL, Stornetta RL, Okusa MD, Guyenet PG (2017) C1 neurons mediate a stress-induced anti-inflammatory reflex in mice. Nat Neurosci 20:700-707

35. Kanbar R, Stornetta RL, Cash DR, Lewis SJ, Guyenet PG (2010) Photostimulation of Phox $2 \mathrm{~b}$ medullary neurons activates cardiorespiratory function in conscious rats. Am J Respir Crit Care Med 182:1184-1194

36. Wenker IC, Abe C, Viar KE, Stornetta DS, Stornetta RL, Guyenet PG (2017) Blood pressure regulation by the rostral ventrolateral medulla in conscious rats: effects of hypoxia, hypercapnia, baroreceptor denervation, and anesthesia. J Neurosci 37:4565-4583

37. McCulloch PF, Panneton WM, Guyenet PG (1999) The rostral ventrolateral medulla mediates the sympathoactivation produced by chemical stimulation of the rat nasal mucosa. J Physiol 516(Pt 2):471-484

38. Guyenet PG, Stornetta RL, Bochorishvili G, Depuy SD, Burke PG, Abbott SB (2013) C1 neurons: the body's EMTs. Am J Physiol Regulat Integr Comp Physiol 305:R187-204 
39. Holstein GR, Friedrich VL Jr, Kang T, Kukielka E, Martinelli GP (2011) Direct projections from the caudal vestibular nuclei to the ventrolateral medulla in the rat. Neuroscience 175:104-117

40. Cai YL, Ma WL, Wang JQ, Li YQ, Li M (2008) Excitatory pathways from the vestibular nuclei to the NTS and the PBN and indirect vestibulo-cardiovascular pathway from the vestibular nuclei to the RVLM relayed by the NTS. Brain Res 1240:96-104
Publisher's Note Springer Nature remains neutral with regard to jurisdictional claims in published maps and institutional affiliations. 